\title{
Conformational States of Polymers Adsorbed on Interfaces
}

\author{
Akira TAKAHASHI \\ Department of Chemistry for Materials, Mie Univeesity, \\ Kamihama-cho, Tsu, Mie 514, Japan
}

(Received November 26, 1990)

\begin{abstract}
Adsorbed layer thickness ( $t$ ) of several dilute polymeric systems were measured by ellipsometry. For physically adsorbed homopolymers, $t$ scales as $M 0.5$ and $\mathrm{M} 0.4$ in theta and good solvents, respectively. Conformational change from "mushroom" to "brush" were observed for a terminally attached polyelectrolyte by adjusting electrostatic "excluded volume". By applying potential for polyelectrolytes adsorbed on a metal surface, both stretched and squeezed conformations were realized. Stiff polymers can be adsorbed either end on or lying flat configurations. Intercalation adsorption into clay lamellas, i.e. slits resulted disk-like conformation. Thus, the concept of "conformation control of adsorbed polymers" was experimentally realized.

KEY WORDS Adsorption / Ellipsometry / Layer Thickness / Conformation / Terminally Attached Chain /
\end{abstract}

Adsorption of polymers on surfaces and interfaces has key roles in a wide variety of fields, such as adhesion, flocculation, stabilization of colloidal particles, reinforcement in composite and biological processes.1 $\sim 4$ The features are quite different from those of adsorption of small molecules. Figure 1 depicts various adsorbed conformations for flexible and stiff or rod-1ike polymers. It should be noted that conformational varieties are more abundant than those in solution.

In the above application fields, it is often necessary to control the adsorbed conformation of polymers to adjust interaction between adsorbed polymer layers. However, before to obtain such control methods, determination of the conformations of adsorbed polymers is one of the most important problems. For determination of conformations, measurements of adsorbed layer thickness and, if possible, segment density distribution normal to the surface are required. In this article, I employed mainly ellipsometry to measure the adsorbed layer thickness and explore conformational control methods experimentally.

\section{RESULTS}


It is now well established that physically adsorbed polymers are held on the surface by relatively few attached segments and the remaining segments from loops and tails. At the theta point, we showed that ellipsometric layer thicknesses $t$ of both polystyrene (PS) 5 and sodium poly (styrene sulfonate) (NaPSS) 7 are proportional to the square root of molecular weight. In good solvent condition, $t$ scales $\mathrm{M} 0.4$ for both PS and NaPSS. Note that the coil radius $\mathrm{Rg}$ in solutions scales M.0.6 Ellipsometric thickness is given by

$t_{e}=\Gamma-1 \int_{0}^{\infty} c(z) z d z \quad(1)$

where $z$ is the distance to the wall (Figure 2), $\Gamma$ is the adsorbance and $C(z)$ is the concentration at the distance $z$. We presented the argument based on de Gennes scaling theory to show that the exponent should be 0.4 in good solvent condition. $6 \sim 9$ That is, the local volume fraction $\phi(z)$ is given by

$$
\phi(z) \simeq \operatorname{voC}(z) \sim(a / z)^{4 / 3} \quad(a<z<R)
$$

where vo is the monomer volume and a is a monomer size. The cut off $R$ is comparable to a random coil size $R \sim a^{3 / 5}$, where $N$ is the degree of polymerization. Inserting (2) into $(1)$, one finds

$$
t_{e}=\int_{0}^{R} \phi(z) z d z \simeq a^{2} / 5
$$

The exponent 0.4 is also observed for the other polymer-solventadsorbent systems as shown in Figure 3, in which surface force apparatus 10 and evanescent wave methodll were used to measure layer thickness. Thus, we may conclude that the physically adsorbed polymer layers have a self-similar structure.

B) Terminally attached chains

Terminally attached chains can be formed by three ways. The first is to prepare polymers whose one end is capped with polar groups. An example is shown in Figure 4(a), in which a very polar 
functional group 1,1 bis ( $\mathrm{p}-\mathrm{N}, \mathrm{N}^{\prime}$-diethy 1 aminopheny l) methanol is attached to polybutadiene ( $T-P B R$ ). The second is the surface grafting and the third method is to employ block copolymers whose one block is preferentially adsorbed on a surface and the other blocks are protruded into solution phase. If the surface density of polymers is low, we have separate "mushrooms", with the coil size $\mathrm{Rg}$, and if the density is high, we have the "brush" as shown in Figure 2(c) and (d).12 In the latter case, the overall thickness of the brush is given by

$L=N a(a / D) 2 / 3 \quad(4)$

where $D$ is the average distance of chains.13 Comparison of the adsorption data of $\mathrm{T}$ $P B R$ with that of a conventional PBR is made in Table I to show the effect of the polar terminal functional group.

The difference in $A$ and the surface coverage of the silanol groups $\theta$ at the silica surface is due to the preferential adsorption of the terminal polar group on $T-P B R$ over that of the butadiene units on PBR.14 16

With respect to carbon black reinforcement, Nagata et al17 have revealed that styrene-butadiene copolymer ( $T-S B R$ ) terminally functionalized with the same group causes a large reduction in carbonto-carbon hysteresis and large increases in resilience and in modulus at high extensions. This study also indicates that instead of

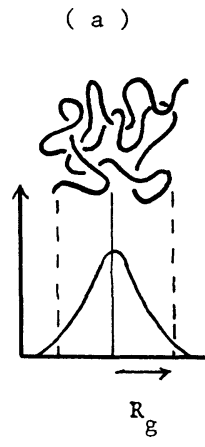

( d )

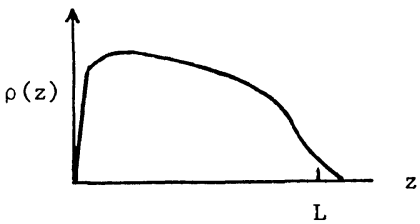

Figure 2. Schematic of the conformations and corresponding segment densities for (a) a random coil in solution, (b) physically adsorbed chain (loop-train-tail); (c,d) terminally attached chains to a wall [(c) mushroom and (d) brush regimes].
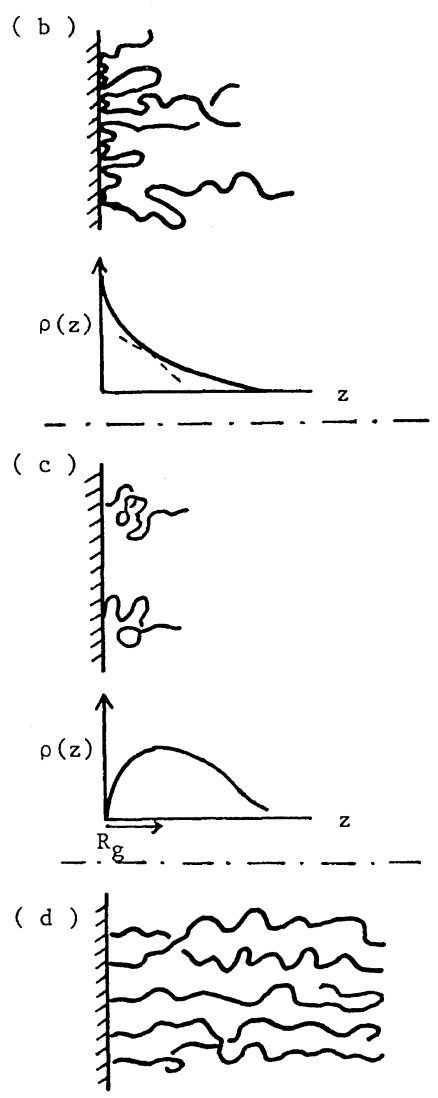


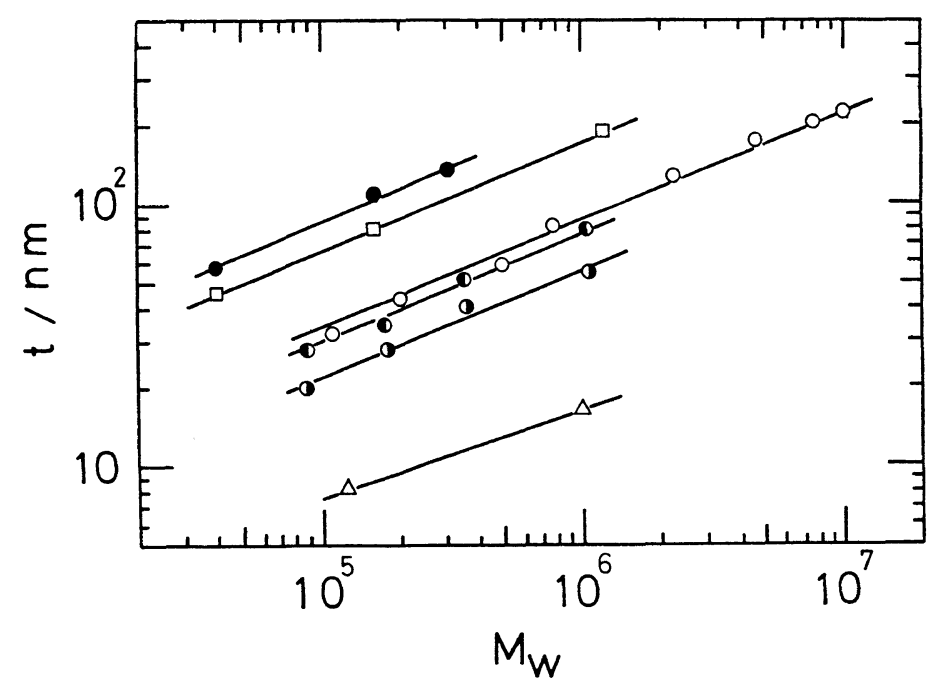

Figure 3. Double logarithmic plot of $t$ against $M$ for several polymer-good solvent-adsorbent systems: polystyrene-CC14-Cr at $35{ }^{\circ} \mathrm{C}, \mathrm{O}$; poly (ethylene oxide)-toluene-Mica at $21^{\circ} \mathrm{C}$, ; PEO$0.1 \mathrm{MKNO}_{3}-\mathrm{Mica}$ at $23^{\circ} \mathrm{C}, \square$; Na poly(styrene sulfonate)-0.1M NaClPt at $250^{\circ} \mathrm{C}$; $\mathrm{NaPSS}-0.5 \mathrm{MNaCl}-\mathrm{Pt}$ at $250^{\circ} \mathrm{C}$; ; poly(methylmethacrylate)-toluene-sapphire at $200^{\circ}, \Delta$. Except PMMA, the lines are drawn with a slope of 0.4 .

covalent bonds, rather salt formation or hydrogen bonding which are labile under stress is very effective for the reinforcement.

The block copolymer which possesses polyelectrolyte blocks as shown in Figure $4(\mathrm{~b})$, has a marked feature that exhibits conformational transition from "mushroom" to "brush" conformations by adjusting electrostatic "excluded volume" interactions between charged groups on the block chains with ionic strength. Figure 5 shows the result of salt concentration dependence of ellipsometric thickness for the ionene-EO-ionene block copolymer adsorbed at the air-aqueous $\mathrm{KBr}$ solution interface.18 With decreasing $\mathrm{KBr}$ concentration, thickness increases from $\mathrm{Rg} \equiv\langle\mathrm{S} 2\rangle 1 / 2$ of the ionene block to the fully stretched (contour) length $\mathrm{L}$, while the $\mathrm{Rg}$ of ionene polyelectrolyte alone never reaches to $\mathrm{L}$ as shown in Figure 5 .

Table I. Adsorption data of $T-P B R$ and PBR on silica at the plateau region at $25^{\circ} \mathrm{C}$ ( $\mathrm{CH}$ : Cyclohexane)

\begin{tabular}{|c|c|c|c|c|c|c|c|}
\hline \multirow{2}{*}{ Sample } & \multirow{2}{*}{$\mathrm{Mn} / 10^{3}$} & \multicolumn{2}{|c|}{$\mathrm{A} \cdot 10^{8}\left(\mathrm{gcm}^{-2}\right)$} & \multicolumn{2}{|c|}{$\theta$} & \multicolumn{2}{|c|}{$\mathrm{p}$} \\
\hline & & $\mathrm{CH}$ & $\mathrm{CC}_{4} 4$ & $\mathrm{CH}$ & $\mathrm{CCl}_{4}$ & $\mathrm{CH}$ & $\mathrm{CCl}_{4}$ \\
\hline $\mathrm{T}-\mathrm{PBR}$ & 222 & 8.5 & 5.9 & 0.65 & 0.26 & 0.25 & 0.12 \\
\hline PBR & 207 & 7.3 & 2.4 & 0.50 & 0.15 & 0.25 & 0.15 \\
\hline
\end{tabular}

*) $\mathrm{p}$ is the fraction of adsorbed monomer units per polymer. 
<smiles>[R7]C(O)(c1ccc(N(CC)CC)cc1)c1ccc(N(CC)CC)cc1</smiles>

( a )

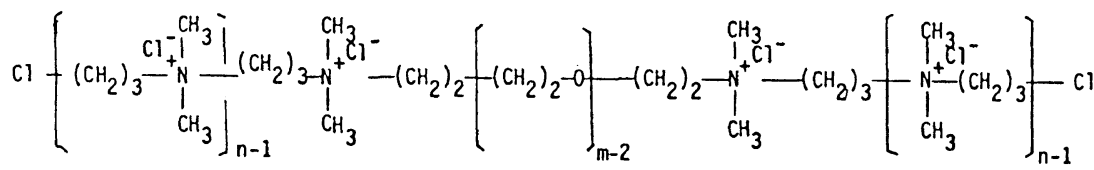

( $\mathrm{b}$ )

Figure 4. (a) Terminally functional poly(butadiene).

(b) 3-3 ione-ethyleneoxide triblock copolymer.

C) Conformational changes of adsorbed polyelectrolyte by applied potential.

Polyelectrolytes, such as sodium salt of poly(acrylic acid), sodium poly(styrene sulfonate)(NaPSS) and deoxyribonucleic acid are adsorbed on metal surfaces. Adsorption of NaPSS from aqueous $\mathrm{NaCl}$ solution onto a platinum plate was studied by ellipsometry.19,20 At rest potential the ellipsometric thickness scales as $\mathrm{M}^{0.5}$ and $\mathrm{M} 0.4$ in theta and good solvents, respectively. By applying potentials, which correspond to cathodic and anodic potential differences relative to the rest potential, we could change adsorbed NaPSS conformation depending on the sign of potentials as shown in Figure 6 and 7 . At the negative potential differences, NaPSS stretches markedly, while at the positive potential differences, it is squeezed probably resulting loop-train conformation.

D) Adsorption of stiff and rodlike polymers

In general, for stiff polymers two principal adsorbed configurations are possible as shown in Figure $1(f)$ and $(g)$. It was shown that both poly- $\gamma$-benzyl-L-glutamate and polyglutamic acid in their helical form adsorb in the standing on end configuration, since their adsorbed layer thicknesses measured by ellipsometry were almost equal to the length of these rodlike polymers 2 .

(Hydroxypropyl) cellulose (HPC) has many OH groups at the ends of side chains, which interact with surface $\mathrm{OH}$ or $\mathrm{O}$ groups of a metal. Moreover, HPC is a liquid crystal forming polymer and is fairly stiff, since its persistent length in water is $18.2 \mathrm{~nm}$. The adsorbed layer thickness of HPC on a platinum foil measured by ellipsometry reveals that the layer thickness is almost $2 \sim 5 \mathrm{~nm}$ irrespective of molecular weight of HPC. Since the width of HPC is $1 \mathrm{~nm}$, and judging from the value of persistent length, HPC behaves intermediate between rodlike molecule and a random coil, the observed thickness indicates somewhat wormlike adsorbed conformation whose height of loops is as high as 5 $\mathrm{nm}$. Thus, HPC adsorbs fairly flat configuration on Pt from water.21 

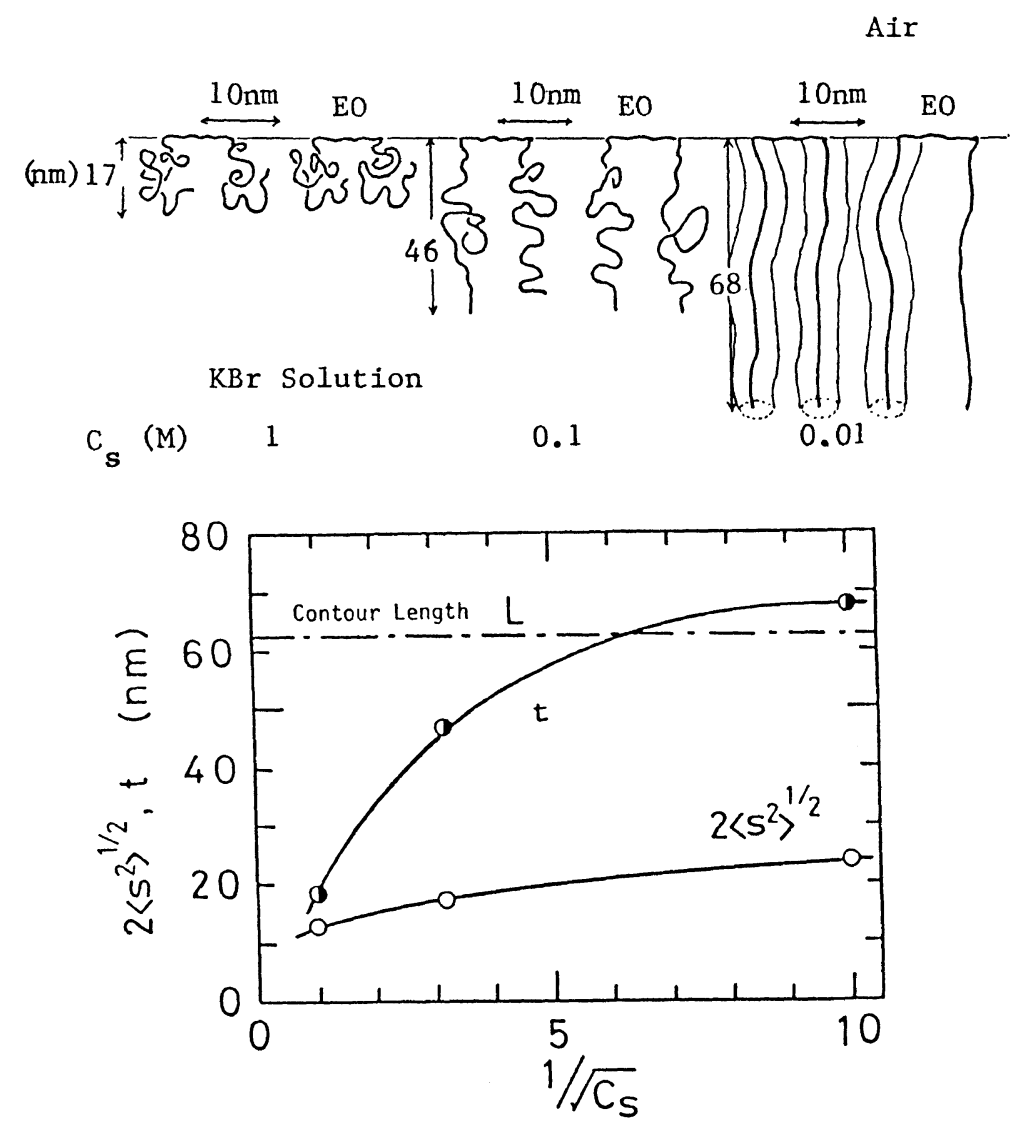

Figure 5. Conformation changes of adsorbed ionene-ethylene oxide block copolymers from "mushroom" regime to "brush" regime. $t$, layer thickness and $\langle s 2>1 / 2$, radius of gyration.

E) Adsorption in various geometries

Since the polymer adsorption on a flat surface is rather exceptional in practice, and adsorption on restricted dimensions as well as various geometries 22,23 is important as shown in Figure 8 . Studies in these directions have been exploited. Here, as an example, I show the polymer adsorption in the slit 24 which is shown in Figure $8(\mathrm{c})$.

Adsorption of poly(ethylene oxid)s (PEO) on calcium montmorillonite (specific surface area $788 \mathrm{~cm}^{2} / \mathrm{g}$ ) from their aqueous solutions was studied. High molecular weight PEO showed high affinity type adsorption isotherm. Concomitant measurement of $d(001)$ spacing of the clay by X-ray diffraction indicates that interlayer spacing is $0.7 \mathrm{~nm}$ when the degree of polymerization $\mathrm{N}$ exceeds $10^{3}$ and is independent of N.26 It was concluded that intercalation adsorption of PEO happened and the adsorbed PEO takes a conformation or form of pancake shaped disk in the interlamellar region, whose radius $R F 2$ was proportional to the square root of $N$, but was smaller than the unperturbed radius of gyration in three dimension as shown in Figure 9 . 


Free energy r of
adsorption of
calculated from the
adsorption isotherm
became more negative with
increase of $\mathrm{N}$ and the
adsorption entropy became
increasingly positive as
shown in Table II. Upon
the intercalation
adsorption of PEO, its
conformation is
restricted and thus
entropy is reduced.
Therefore, the positive
entropy originates from
the desorption of water
molecules from the
interlamella
region. $25 \sim 27$ a

Free energy of adsorption of PEO calculated from the became more negative with increase of $\mathrm{N}$ and the adsorption entropy became increasingly positive as shown in Table II. Upon the intercalation adsorption of PEO, its conformation is entropy is reduced. Therefore, the positive entropy originates from the desorption of water inter lamella region. $25 \sim 27$

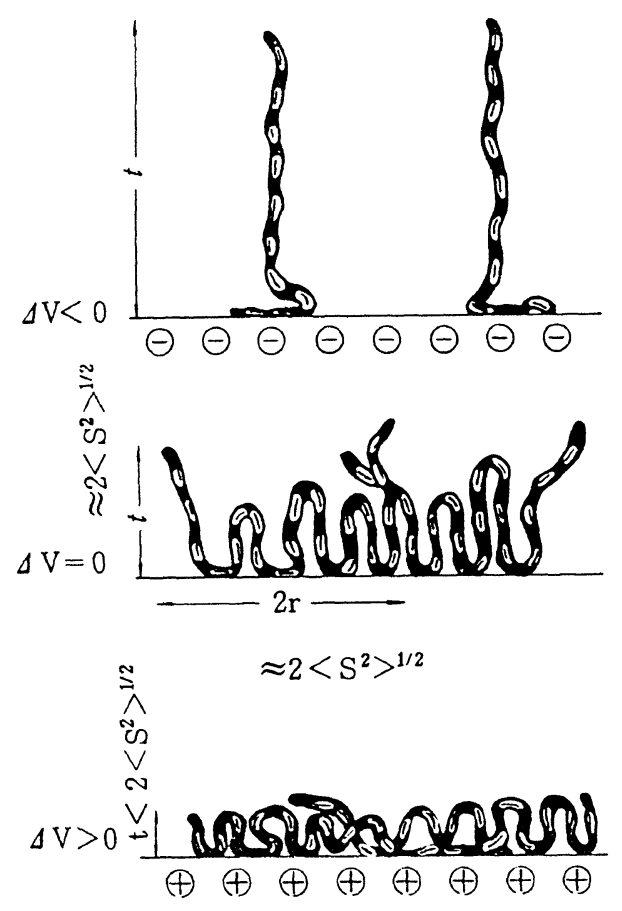

Figure 6. Models for the conformation changes of adsorbed NaPSS chains under potential variation.

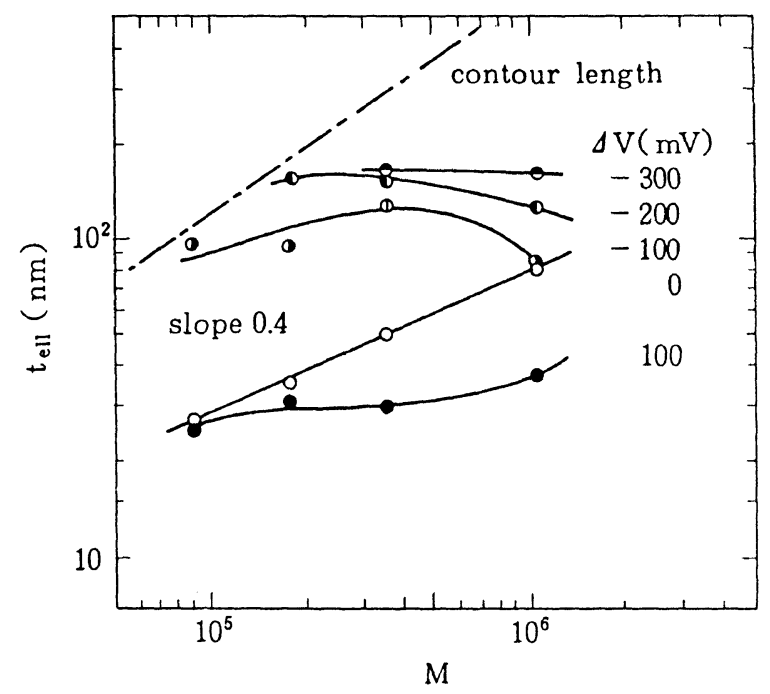

Figure 7. Double logarithmic plot of adsorbed layer thickness versus molecular weight of NaPSS in aqueous $0.1 \mathrm{M} \mathrm{NaCl}$ solution. 


\section{CONCLUSIONS}

I could realize various adsorbed conformations and configurations of polymers by desigining polymer structure and controlling interactions among polymers, solvents and adsorbents. In Figure 10, the expoent $v$ of molecular weight dependence of the adsorbed layer thickness is summarized for various adsorbed conformations.

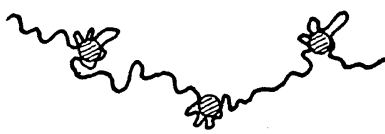

( a )

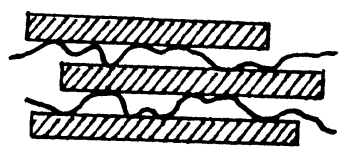

( c)

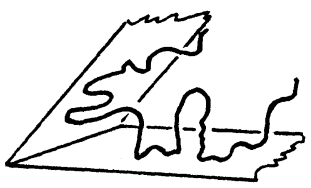

( e )

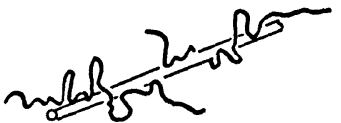

( $\mathrm{b}$ )

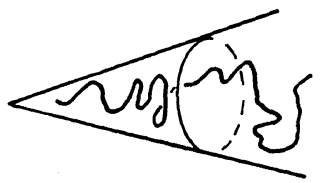

( d )

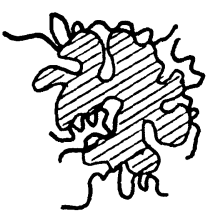

( $f$ )

Figure 8. Adsorbed polymer chains in various geometries.

(a) necklace type adsorption on colloidal sphere;

(b) on a rod; (c) in slits; (d) in a cone; (e) in a wedge;

(f) on a fractal surface.

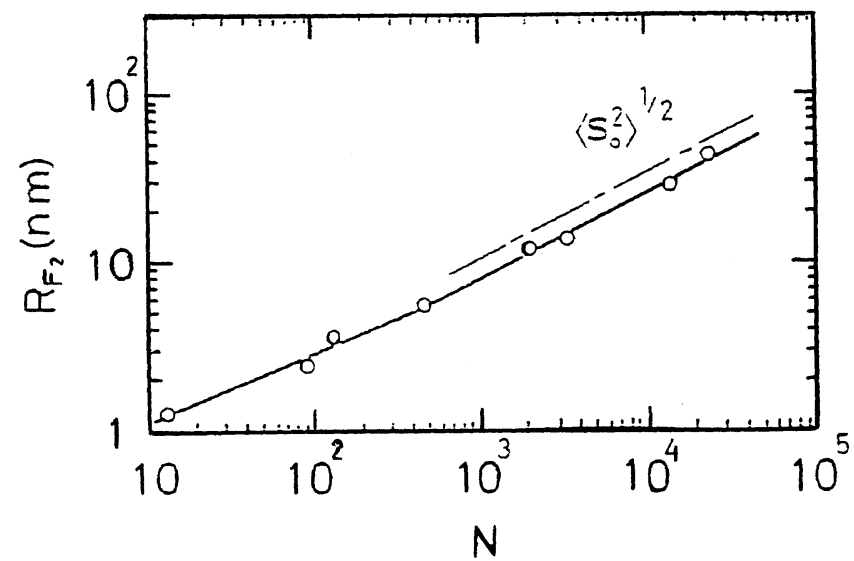

Figure 9. Chain length dependence of radius of adsorbed PEO disk $\left(R_{F_{2}}\right)$ and unperturbed radius of gyration. 
Table II. Non-standard thermodynamic data for the adsorption of PEO by calcium montmorillionite

\begin{tabular}{|c|c|c|c|c|}
\hline \multirow[b]{2}{*}{$M_{W}$ of $P E O$} & \multicolumn{2}{|l|}{$15^{\circ} \mathrm{C}$} & \multicolumn{2}{|l|}{$35^{\circ} \mathrm{C}$} \\
\hline & $\begin{array}{c}-\Delta \mathrm{Gm} \\
\mathrm{cal} / \mathrm{mol}\end{array}$ & $\begin{array}{c}-\Delta \mathrm{Gm} \\
\mathrm{cal} / \mathrm{mol}\end{array}$ & $\begin{array}{c}-\Delta \mathrm{H}^{\mathrm{m}} \\
\mathrm{cal} / \mathrm{mol}\end{array}$ & $\begin{array}{l}\Delta s \mathrm{~s} \\
\mathrm{e} \cdot \mathrm{u} .\end{array}$ \\
\hline 400 & 783 & 821 & 716 & 0.34 \\
\hline 6,000 & 3385 & 3621 & 1102 & 7.9 \\
\hline 20,000 & 4698 & 5140 & 1674 & 11.5 \\
\hline
\end{tabular}

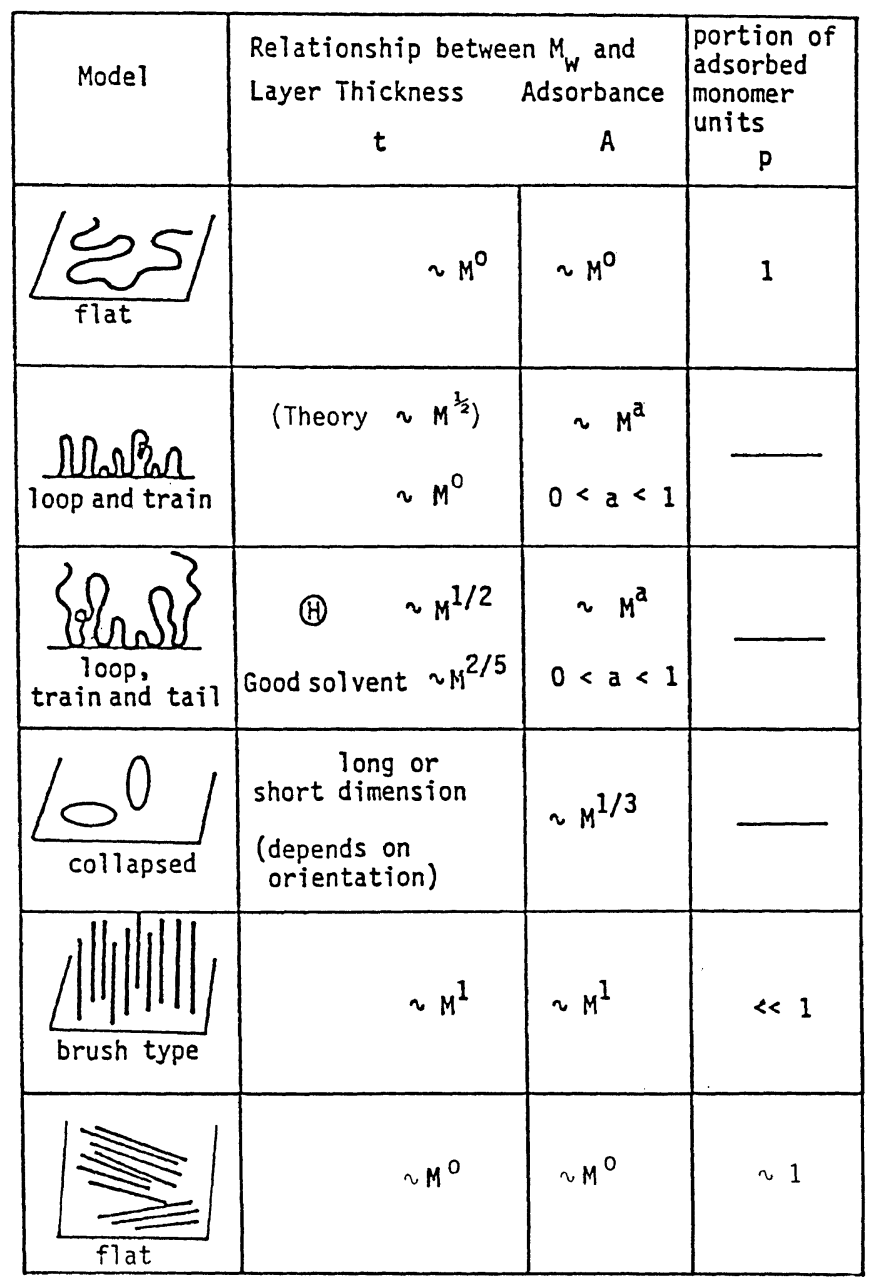

Figure 10. Models for the conformation of adsorbed polymers. 
Acknowledgment

I wish to thank my collaborators on the research of polymer adsorption, especially Professors Masami Kawaguchi and Tadaya Kato.

\section{REFERENCES}

1. A. Takahashi and M. Kawaguchi, Adv. Polymer Sci., 46, 1 (1982).

2. A. Takahashi, in "Molecular Conformation and Dynamics of Macromolecules in Condensed Systems", M. Nagasawa ed., Elsevier, Amsterdam, 1988, pp. 353369.

3. A. Takahashi, in "Frontiers of Macromolecular Science", T. Saegusa, T. Higashimura and A. Abe eds., Blackwell, London, 1989, pp. 331336 .

4. D. H. Napper, "Polymric Stabilization of Colloidal Dispersions", Academic, London, (1983).

5. A. Takahashi, M. Kawaguchi, H. Hirota and T. Kato, Macromolecules, 13, 884 (1980).

6. M. Kawaguchi and A. Takahashi, Macromolecules, 16, 1465 (1983).

7. M. Kawaguchi, K. Hayashi and A. Takahashi, Macromolecules, 17, 2066 (1984).

8. P. G. de Gennes, Macromolecules, 14, 1637 (1981); 15, 492 (1982).

9. P. G. de Gennes, "Scaling Concepts in Polymer Physics", Cornell Univ. Press. Ithaca, N. Y., 2nd printing (1985).

10. J. Klein and P. F. Luckham, Macromolecules, 19, 2007 (1986).

11. I. Caucheteux, H. Hervet, R. Jerome and F. Rondelez, J. C. S. Faraday Trans., 86, 1369 (1990).

12. S. Alexander, J. Physique (Paris), 38, 1069 (1980).

13. P. G. de Gennes, Macromolecules, 13, 1069 (1980).

14. M. Kawaguchi, M. Kawarabayashi, N. Nagata, T. Kato, A Yoshioka and A. Takahashi, Macromolecules, 21, 1059 (1988).

15. M. Kawaguchi, H. Hanai, A. Takahashi, N. Nagata and A. Yoshioka, Colloids and Surfaces, 37, 319 (1989).

16. M. Kawaguchi, M. Kawarabayashi, A. Takahashi, N. Nagata, and A. Yoshioka, Colloids and Surfaces, 48, 363 (1990).

17. N. Nagata, T. Kobatake, H. Watanabe, A. Ueda and A. Yoshioka, Rubber Chem. Technol., 60, 837 (1987).

18. M. Kawaguchi, M. Oohira, M. Tajima and A. Takahashi, Polymer J., 12, 849 (1980).

19. M. Kawaguchi, K. Hayashi and A. Takahashi, Macromolecules, 21, 1016 (1988).

20. M. Kawaguchi, K. Hayashi and A. Takahashi, Colloids and Surfaces, 31, 73 (1988).

21. H. Kubo and A. Takahashi, in preparation.

22. P. A. Pincus, C. J. Sandroff and T. A. Witten, J. Physique (Paris), 45, 725 (1984).

23. Z-G. Wang, A. M. Nemerovsky and K. F. Freed, J. Chem., Phys., 86, 4266 (1987).

24. M. Daoud and P. G. de Gennes, J. Physique (Paris), 38, 85 (1977).

25. B. K. G. Theng, "Formation and Properties of Clay-Polymer Complexes", Elsevier, Amsterdam. (1979).

26. A. Kobayashi, M. Kawaguchi, T. Kato and A. Takahashi, Bull. Inst. Chem. Res. Kyoto University, 66, 176 (1988).

27. R. L. Parfitt and D. J. Greenland, Clay Minerals, 8, 305 (1970). 